\title{
FLUCTUATIONS OF THE NUMBER OF CRITICAL POINTS OF RANDOM TRIGONOMETRIC POLYNOMIALS*
}

BY

\author{
LIVIU I. NICOLAESCU
}

\begin{abstract}
Denote by $Z_{\nu}$ the number of critical points of a random trigonometric polynomial of degree $\leq \nu$. We prove that as $\nu \rightarrow \infty$ the expectation of $Z_{\nu}$ is asymptotic to $2 \nu \sqrt{\frac{3}{5}}$ while its variance is asymptotic to $\delta_{\infty} \nu$ where $\delta_{\infty} \approx 0.35$.

Mathematics Subject Classification 2010: 33B10, 42A61, 60D99.

Key words: random functions, critical points, Kac-Rice formula.
\end{abstract}

\section{Introduction}

A random trigonometric polynomial of degree $\leq \nu$ is a trigonometric polynomial of the form

$$
\eta_{\nu}(t)=\frac{1}{\sqrt{2 \pi}} a_{0}+\frac{1}{\sqrt{\pi \nu}} \sum_{m=1}^{\nu}\left(a_{m} \cos m t+b_{m} \sin m t\right), \quad t \in \mathbb{R} / 2 \pi \mathbb{Z},
$$

where $a_{m}, b_{m}$ are independent normally distributed random variables with mean 0 and variance 1 .

The number $Z_{\nu}$ of critical points of such a random polynomial is itself a random variable. The goal of this paper is to describe two important statistical quantities associated to this random quantity, namely its expectation, $E_{\nu}$, and its variance, $V_{\nu}$. We are particularly interested in the behavior of these quantities as the degree $\nu$ goes to infinity. In probabilistic parlance, $\eta_{\nu}$ is a (stationary) Gaussian process on the unit circle $\mathbb{R} / 2 \pi \mathbb{Z}$ and as $\nu \rightarrow \infty$ this process approaches the singular process called white noise.

\footnotetext{
*Invited paper
} 
The main result of this paper shows that there exist (explicit) positive constants $c_{1}, c_{2}$ such that

$$
E_{\nu} \sim c_{1} \nu, \quad V_{\nu} \sim c_{2} \nu \text { as } \nu \rightarrow \infty
$$

The above estimates imply an interesting concentration phenomenon. Suppose that we ask what are the odds that the number of critical points of a random trigonometric polynomial differs by more than $1 \%$ from the expected number of critical points. In other words we ask what is the probability of the event

$$
\left|Z_{\nu}-E_{\nu}\right| \geq \frac{E_{\nu}}{100}
$$

The Chebyshev inequality shows

$$
P\left(\left|Z_{\nu}-E_{\nu}\right| \geq 0.1 E_{\nu}\right) \leq \frac{V_{\nu}}{\left(0.01 E_{\nu}\right)^{2}} \sim \frac{10^{4} c_{2}}{c_{1}^{2} \nu} \text { as } \nu \rightarrow \infty .
$$

Thus, the above event is highly unlikely if the degree $\nu$ is very large. This indicates that the highly oscillatory parts of a random trigonometric polynomials have a dominant effect in the creation of critical points. This is reminiscent of the classical Poincaré phenomenon: the area of a large dimensional round sphere tends to be concentrated on a narrow tube around an Equator ${ }^{1}$ of fixed and small codimension.

The statistics of the critical set of the above random function is identical to the statistics of the zero set of the random function

$$
\xi_{\nu}(t)=\frac{d \eta_{\nu}((t)}{d t}=\frac{1}{\sqrt{\pi \nu}} \sum_{m=1}^{\nu}\left(-m a_{m} \sin m t+m b_{m} \cos m t\right) .
$$

Equivalently, consider the rescaled random function

$$
\Phi_{\nu}(t)=\frac{1}{\sqrt{\pi \nu^{3}}} \sum_{m=1}^{n}\left(m c_{m} \cos \left(\frac{t}{\nu}\right)+m d_{m} \sin \left(\frac{t}{\nu}\right)\right), t \in[-\pi \nu, \pi \nu],
$$

where $c_{m}, d_{m}$ are independent random variables with identical standard normal distribution, and the random variable

$$
Z_{\nu}:=\text { the number of zeros of } \Phi_{\nu}(t) \text { in the interval }[-\pi \nu, \pi \nu] \text {. }
$$

\footnotetext{
${ }^{1}$ By Equator on a sphere centered at the origin of an Euclidean space we mean the intersection of the sphere with a vector subspace.
} 
Note that the expectation of $Z_{\nu}$ is precisely the expected number of critical points of a random trigonometric polynomial in the space

$$
\boldsymbol{T}_{\nu}:=\operatorname{span}\{\cos (m \theta), \sin (m \theta) ; m=0, \ldots \nu\},
$$

equipped with the inner product

$$
(u, v)=\int_{0}^{2 \pi} u(\theta) v(\theta) d \theta,
$$

and Gaussian measure

$$
d \boldsymbol{\gamma}(u)=\frac{1}{(2 \pi)^{\frac{\operatorname{dim} \boldsymbol{T}_{\nu}}{2}}} e^{-\frac{\|u\|^{2}}{2}} d u .
$$

We let $\boldsymbol{E}(\zeta)$, and respectively $\boldsymbol{v} \boldsymbol{a r}(\zeta)$, denote the expectation, and respectively the variance, of a random variable $\zeta$. The random function $\Phi_{\nu}$ is a stationary Gaussian process with covariance kernel

$$
R_{\nu}(t)=\boldsymbol{E}\left(\Phi_{\nu}(t) \Phi_{\nu}(0)\right)=\frac{1}{\pi \nu^{3}} \sum_{m=1}^{\nu} m^{2} \cos \left(\frac{m t}{\nu}\right) .
$$

The Rice formula, [2, Eq. (10.3.1)], implies that

$$
\boldsymbol{E}\left(Z_{\nu}\right)=2 \nu\left(\frac{\lambda_{2}(\nu)}{\lambda_{0}(\nu)}\right)^{\frac{1}{2}},
$$

where

$$
\lambda_{0}(\nu)=R_{\nu}(0)=\frac{1}{\pi \nu^{3}} \sum_{m=1}^{\nu} m^{2} \text { and } \lambda_{2}(\nu)=-R_{\nu}^{\prime \prime}(0)=\frac{1}{\pi \nu^{5}} \sum_{m=1}^{\nu} m^{4} .
$$

This proves that

$$
\boldsymbol{E}\left(Z_{\nu}\right) \sim 2 n \sqrt{\frac{3}{5}} \text { as } n \rightarrow \infty .
$$

The following is the main result of this paper.

Theorem 1.1. Set

$$
\bar{\lambda}_{0}:=\lim _{\nu \rightarrow \infty} \lambda_{0}(\nu)=\frac{1}{3}, \quad \bar{\lambda}_{2}:=\lim _{\nu \rightarrow \infty} \lambda_{2}(\nu):=\frac{1}{5} .
$$


Then for any $t \in \mathbb{R}$ the limit $\lim _{\nu \rightarrow \infty} R_{\nu}(t)$ exists, it is equal to

$$
R_{\infty}(t):=\frac{1}{t^{3}} \int_{0}^{t} \tau^{2} \cos \tau d \tau=\int_{0}^{1} \lambda^{2} \cos (\lambda t) d \lambda, \quad \forall t \in \mathbb{R}
$$

and

$$
\lim _{\nu \rightarrow \infty} \frac{1}{\nu} \operatorname{var}\left(Z_{\nu}\right)=\delta_{\infty}:=\frac{2}{\pi} \int_{-\infty}^{\infty}\left(f_{\infty}(t)-\frac{\bar{\lambda}_{2}}{\bar{\lambda}_{0}}\right) d t+2 \sqrt{\frac{\bar{\lambda}_{2}}{\bar{\lambda}_{0}},}
$$

where

$$
f_{\infty}(t)=\frac{\left(\bar{\lambda}_{0}^{2}-R_{\infty}^{2}\right) \bar{\lambda}_{2}-\bar{\lambda}_{0}\left(R_{\infty}^{\prime}\right)^{2}}{\left(\lambda_{0}^{2}-R_{\infty}^{2}\right)^{\frac{3}{2}}}\left(\sqrt{1-\rho_{\infty}^{2}}+\rho_{\infty} \arcsin \rho_{\infty}\right),
$$

and

$$
\rho_{\infty}=\frac{R_{\infty}^{\prime \prime}\left(\bar{\lambda}_{0}^{2}-R_{\infty}^{2}\right)+\left(R_{\infty}^{\prime}\right)^{2} R_{\infty}}{\left(\bar{\lambda}_{0}^{2}-R_{\infty}^{2}\right) \bar{\lambda}_{2}-\bar{\lambda}_{0}\left(R_{\infty}^{\prime}\right)^{2}}
$$

Moreover, the constant $\delta_{\infty}$ is positive. ${ }^{2}$

We should perhaps put this result in some perspective. Recently, GRANVILLE and Wigman [4] have investigated the behavior of the number $N_{\nu}$ of zeros of a random trigonometric polynomial of the form

$$
P_{\nu}(t)=\frac{1}{\sqrt{\pi \nu}} \sum_{m=1}^{\nu}\left(a_{m} \cos m t+b_{m} \sin m t\right), \quad t \in[0,2 \pi),
$$

where $a_{m}, b_{m}$ are independent normally distributed random variables with mean 0 and variance 1 . It is known that (see $[3,6]$ )

$$
\boldsymbol{E}\left(N_{\nu}\right) \sim \sqrt{\frac{2}{3}} \nu \text { as } \nu \rightarrow \infty,
$$

but the authors show that a much more precise result is true. More precisely, they prove that there exists $c>0$ such that as $\nu \rightarrow \infty$ the random variable

$$
\widetilde{N}_{\nu}=\frac{1}{\sqrt{c \nu}}\left(N_{\nu}-\boldsymbol{E}\left(N_{\nu}\right)\right)
$$

${ }^{2}$ Numerical experiments indicate that $\delta_{\infty} \approx 0.35$. 
converges to the standard Gaussian. Our strategy is inspired from [4] and we believe that a central limit theorem is valid in the case of the statistics of critical points as well. We plan to pursue this aspect elsewhere.

Let us observe that the space of trigonometric polynomials of degree $\leq \nu$ can be identified with the space $\boldsymbol{U}_{L}$ spanned by the eigenfunctions of the Laplacian of $S^{1}$ corresponding to eigenvalues $\leq L:=\nu^{2}$. The estimate (1.1) can be rewritten as

$$
\boldsymbol{E}\left(Z_{\nu}\right) \sim \sqrt{\frac{3}{5}} \operatorname{dim} \boldsymbol{U}_{\nu^{2}} \text { as } \nu \rightarrow \infty .
$$

In [5] we proved a higher dimensional generalization of this estimate. More precisely we have shown that for any $m>0$ there exists an explicit positive constant $C_{m}>0$ such that for any compact, connected, smooth $m$-dimensional Riemann manifold if we denote by $Z_{L}$ the number of critical points of a random linear combination of eigenfunctions of the Laplacian $\Delta_{g}$ corresponding to eigenvalues $\leq L$, then

$$
\boldsymbol{E}\left(Z_{L}\right) \sim C_{m} \operatorname{dim} \boldsymbol{U}_{L} \text { as } L \rightarrow \infty .
$$

Theorem 1.1 suggests the following higher dimensional question: does there exist a constant $c>1$, possibly dependent on $M$ and $g$, such that

$$
\frac{1}{c} \operatorname{dim} \boldsymbol{U}_{L}<\boldsymbol{v a r}\left(Z_{L}\right)<c \operatorname{dim} \boldsymbol{U}_{L} \text { as } L \rightarrow \infty \text { ?. }
$$

\section{Proof of the main theorem}

We follow a strategy inspired from [4]. The variance of $Z_{\nu}$ can be computed using the results in $[2, \S 10.6]$. We introduce the Gaussian field

$$
\boldsymbol{\Psi}\left(t_{1}, t_{2}\right):=\left[\begin{array}{c}
\Phi_{\nu}\left(t_{1}\right) \\
\Phi_{\nu}\left(t_{2}\right) \\
\Phi_{\nu}^{\prime}\left(t_{1}\right) \\
\Phi_{\nu}^{\prime}\left(t_{2}\right)
\end{array}\right]
$$

Its covariance matrix depends only on $t=t_{2}-t_{1}$. We have (compare with $[4$, Eq. (17)])

$$
\boldsymbol{\Xi}(t)=\left[\begin{array}{cccc}
\lambda_{0} & R_{\nu}(t) & 0 & R_{\nu}^{\prime}(t) \\
R_{\nu}(t) & \lambda_{0} & -R_{\nu}^{\prime}(t) & 0 \\
0 & -R_{\nu}^{\prime}(t) & \lambda_{2} & -r_{\nu}^{\prime \prime}(t) \\
R_{\nu}^{\prime}(t) & 0 & -R_{\nu}^{\prime \prime}(t) & \lambda_{2}
\end{array}\right]=:\left[\begin{array}{cc}
A & B \\
B^{\dagger} & C
\end{array}\right] .
$$


As explained in [6], to apply $[2, \S 10.6]$ we only need that $\boldsymbol{\Xi}(t)$ is nondegerate. This is established in the next result whose proof can be found in Appendix A.

Lemma 2.1. The matrix $\boldsymbol{\Xi}(t)$ is nonsingular if and only if $t \notin 2 \pi \nu \mathbb{Z}$.

For any vector

$$
\boldsymbol{x}:=\left[\begin{array}{l}
x_{1} \\
x_{2} \\
x_{3} \\
x_{4}
\end{array}\right] \in \mathbb{R}^{4}
$$

we set

$$
p_{t_{1}, t_{2}}(\boldsymbol{x}):=\frac{1}{4 \pi^{2}(\operatorname{det} \boldsymbol{\Xi})^{1 / 2}} e^{-\frac{1}{2}\left(\boldsymbol{\Xi}^{-1} \boldsymbol{x}, \boldsymbol{x}\right)} .
$$

Then, the results in $[2, \S 10.6]$ show that

$$
\boldsymbol{E}\left(Z_{\nu}^{2}\right)-\boldsymbol{E}\left(Z_{\nu}\right)=\int_{\boldsymbol{I}_{\nu} \times \boldsymbol{I}_{\nu}}\left(\int_{\mathbb{R}^{2}}\left|y_{1} y_{2}\right| \cdot p_{t_{1}, t_{2}}\left(0,0, y_{1}, y_{2}\right)\left|d y_{1} d y_{2}\right|\right)\left|d t_{1} d t_{2}\right| .
$$

As in [4] we have

$$
\mathbf{\Xi}(t)^{-1}=\left[\begin{array}{cc}
* & * \\
* & \Omega^{-1}
\end{array}\right], \Omega=C-B^{\dagger} A^{-1} B .
$$

More explicitly,

$$
\begin{aligned}
\Omega & =C-B^{\dagger} A^{-1} B \\
& =\left[\begin{array}{cc}
\lambda_{2} & -R_{\nu}^{\prime \prime}(t) \\
-R_{\nu}^{\prime \prime}(t) & \lambda_{2}
\end{array}\right] \\
& -\frac{1}{\lambda_{0}^{2}-R_{\nu}(t)^{2}}\left[\begin{array}{cc}
0 & -R_{\nu}^{\prime}(t) \\
R_{\nu}^{\prime}(t) & 0
\end{array}\right] \cdot\left[\begin{array}{cc}
\lambda_{0} & -R_{\nu}(t) \\
-R_{\nu}(t) & \lambda_{0}
\end{array}\right] \cdot\left[\begin{array}{cc}
0 & R_{\nu}^{\prime}(t) \\
-R_{\nu}^{\prime}(t) & 0
\end{array}\right] \\
& =\left[\begin{array}{cc}
\lambda_{2} & -R_{\nu}^{\prime \prime} \\
-R_{\nu}^{\prime \prime} & \lambda_{2}
\end{array}\right]-\frac{\left(R_{\nu}^{\prime}\right)^{2}}{\lambda_{0}^{2}-R_{\nu}^{2}}\left[\begin{array}{cc}
\lambda_{0} & R_{\nu} \\
R_{\nu} & \lambda_{0}
\end{array}\right]=\mu\left[\begin{array}{cc}
1 & -\rho \\
-\rho & 1
\end{array}\right],
\end{aligned}
$$

where

$$
\mu=\mu_{\nu}=\frac{\left(\lambda_{0}^{2}-R_{\nu}^{2}\right) \lambda_{2}-\lambda_{0}\left(R_{\nu}^{\prime}\right)^{2}}{\lambda_{0}^{2}-R_{\nu}^{2}}, \rho=\rho_{\nu}=\frac{R_{\nu}^{\prime \prime}\left(\lambda_{0}^{2}-R_{\nu}^{2}\right)+\left(R_{\nu}^{\prime}\right)^{2} R_{\nu}}{\left(\lambda_{0}^{2}-R_{\nu}^{2}\right) \lambda_{2}-\lambda_{0}\left(R_{\nu}^{\prime}\right)^{2}} .
$$

We want to emphasize that in the above equalities the constants $\lambda_{0}$ and $\lambda_{2}$ do depend on $\nu$, although we have not indicated this in our notation. 
Remark 2.2. The nondegeneracy of $\boldsymbol{\Xi}$ implies that $\mu_{\nu}(t) \neq 0$ and $\left|\rho_{\nu}(t)\right|<1$, for all $t \notin 2 \pi \nu \mathbb{Z}$.

We obtain as in [4, Eq. (24)]

$$
\operatorname{det} \boldsymbol{\Xi}=\operatorname{det} A \cdot \operatorname{det} \Omega=\mu^{2}\left(\lambda_{0}^{2}-R_{\nu}^{2}\right)\left(1-\rho^{2}\right), \quad \Omega^{-1}=\frac{1}{\mu\left(1-\rho^{2}\right)}\left[\begin{array}{ll}
1 & \rho \\
\rho & 1
\end{array}\right] .
$$

We can now rewrite the equality $(2.1)$ as $\left(t=t_{2}-t_{1}\right)$

$$
\begin{aligned}
& \boldsymbol{E}\left(Z_{\nu}^{2}\right)-\boldsymbol{E}\left(Z_{\nu}\right) \\
& =\int_{\boldsymbol{I}_{\nu} \times \boldsymbol{I}_{\nu}}\left(\int_{\mathbb{R}^{2}}\left|y_{1} y_{2}\right| e^{-\frac{y_{1}^{2}+2 \rho y_{1} y_{2}+y_{2}^{2}}{2 \mu\left(1-\rho^{2}\right)}} \frac{\left|d y_{1} d y_{2}\right|}{4 \pi^{2}}\right) \frac{\left|d t_{1} d t_{2}\right|}{\mu \sqrt{\left(\lambda_{0}^{2}-R_{\nu}^{2}\right)\left(1-\rho^{2}\right)}} \\
& =\int_{\boldsymbol{I}_{\nu} \times \boldsymbol{I}_{\nu}}\left(\int_{\mathbb{R}^{2}}\left|y_{1} y_{2}\right| e^{-\frac{y_{1}^{2}+2 \rho y_{1} y_{2}+y_{2}^{2}}{2\left(1-\rho^{2}\right)}} \frac{\left|d y_{1} d y_{2}\right|}{4 \pi^{2}}\right) \frac{\mu\left|d t_{1} d t_{2}\right|}{\sqrt{\left(\lambda_{0}^{2}-R_{\nu}^{2}\right)\left(1-\rho^{2}\right)}} .
\end{aligned}
$$

From [1, Eq. (A.1)] we deduce that

$$
\int_{\mathbb{R}^{2}}\left|y_{1} y_{2}\right| e^{-\frac{y_{1}^{2}+2 \rho y_{1} y_{2}+y_{2}^{2}}{2\left(1-\rho^{2}\right)}} \frac{\left|d y_{1} d y_{2}\right|}{4 \pi^{2}}=\frac{1-\rho^{2}}{\pi^{2}}\left(1+\frac{\rho}{\sqrt{1-\rho^{2}}} \arcsin \rho\right) .
$$

Hence

$$
\begin{gathered}
\boldsymbol{E}\left(Z_{\nu}^{2}\right)-\boldsymbol{E}\left(Z_{\nu}\right)=\frac{1}{\pi^{2}} \int_{\boldsymbol{I}_{\nu} \times \boldsymbol{I}_{\nu}} \frac{\mu}{\left(\lambda_{0}^{2}-R_{\nu}^{2}\right)^{\frac{1}{2}}}\left(\sqrt{1-\rho^{2}}+\rho \arcsin \rho\right)\left|d t_{1} d t_{2}\right| \\
(2.2)=\frac{1}{\pi^{2}} \int_{\boldsymbol{I}_{\nu} \times \boldsymbol{I}_{\nu}} \underbrace{\frac{\left(\lambda_{0}^{2}-R_{\nu}^{2}\right) \lambda_{2}-\lambda_{0}\left(R_{\nu}^{\prime}\right)^{2}}{\left(\lambda_{0}^{2}-R_{\nu}^{2}\right)^{\frac{3}{2}}}\left(\sqrt{1-\rho^{2}}+\rho \arcsin \rho\right)}_{=: f_{\nu}(t)}\left|d t_{1} d t_{2}\right| .
\end{gathered}
$$

The function $f_{\nu}(t)=f_{\nu}\left(t_{2}-t_{1}\right)$ is doubly periodic with periods $2 \pi \nu, 2 \pi \nu$ and we conclude that

$$
\boldsymbol{E}\left(\left[Z_{\nu}\right]_{2}\right):=\boldsymbol{E}\left(Z_{\nu}^{2}\right)-\boldsymbol{E}\left(Z_{\nu}\right)=\frac{2 \nu}{\pi} \int_{-\pi \nu}^{\pi \nu} f_{\nu}(t) d t .
$$

We conclude that

$$
\begin{aligned}
\boldsymbol{v a r}\left(Z_{\nu}\right) & =\boldsymbol{E}\left(\left[Z_{\nu}\right]_{2}\right)+\boldsymbol{E}\left(Z_{\nu}\right)-\left(\boldsymbol{E}\left(Z_{\nu}\right)\right)^{2}=\boldsymbol{E}\left(\left[Z_{\nu}\right]_{2}\right)-\left[\boldsymbol{E}\left(Z_{\nu}\right)\right]_{2} \\
& =\frac{2 \nu}{\pi} \int_{-\pi \nu}^{\pi \nu}\left(f_{\nu}(t)-\frac{\lambda_{2}}{\lambda_{0}}\right) d t+2 \nu \sqrt{\frac{\lambda_{2}}{\lambda_{0}}}
\end{aligned}
$$


To complete the proof of Theorem 1.1 we need to investigate the integrand in (2.3). This requires a detailed understanding of the behavior of $R_{\nu}$ as $\nu \rightarrow \infty$. It is useful to consider more general sums of the form

$$
A_{\nu, r}(t)=\frac{1}{\nu^{r+1}} \sum_{m=1}^{\nu} m^{r} \cos \frac{m t}{\nu}, \quad B_{\nu, r}(t)=\frac{1}{\nu^{r+1}} \sum_{m=1}^{\nu} m^{r} \sin \frac{m t}{\nu}, r \geq 1 .
$$

Note that if we set $z:=\cos \frac{m t}{\nu}+\boldsymbol{i} \sin \frac{m t}{\nu}$. We have

$$
A_{\nu, r}(t)+i B_{\nu, r}(t)=\frac{1}{\nu^{r+1}} \underbrace{\sum_{m=1}^{\nu} m^{r} z^{m}}_{=: C_{\nu, r}(t)} .
$$

Observe that

$$
R_{\nu}^{(k)}(t)=-\frac{1}{\pi \nu^{k+3}} \mathbf{R e}\left(\frac{1}{\boldsymbol{i}^{k+2}} C_{\nu, k+2}(t)\right)
$$

We set

$$
A_{\infty, r}(t)=\frac{1}{t^{r+1}} \int_{0}^{t} \tau^{r} \cos \tau d \tau, \quad B_{\infty, r}(t):=\frac{1}{t^{r+1}} \int_{0}^{t} \tau^{r} \sin \tau d \tau .
$$

Observe that $R_{\nu}=A_{\nu, 2}$ and $R_{\infty}=A_{\infty, 2}$. We have the following result.

\section{Lemma 2.3.}

$$
\left|A_{\nu, r}(t)-A_{\infty, r}(t)\right|+\left|B_{\nu, r}(t)-B_{\infty, r}(t)\right|=O\left(\frac{\max (1, t)}{\nu}\right), \forall t \geq 0
$$

where, above and in the sequel, the constant implied by the O-symbol is independent of $t$ and $\nu$. In particular

$$
\lim _{\nu \rightarrow \infty} \frac{1}{\nu^{r+1}} C_{\nu, r}(t)=C_{r}(t):=\frac{1}{t^{r+1}} \int_{0}^{t} \tau^{r} e^{i \tau} d \tau, \quad \forall t \geq 0
$$

Proof. We have

$$
A_{\nu}(t)=\frac{1}{\nu^{r+1}} \sum_{m=1}^{\nu} m^{r} \cos \left(\frac{m t}{\nu}\right)=\frac{1}{t^{r+1}} \underbrace{\sum_{m=1}^{\nu}\left\{\left(\frac{m t}{\nu}\right)^{r} \cos \left(\frac{m t}{\nu}\right)\right\} \cdot\left(\frac{t}{\nu}\right)}_{=: S_{\nu}(t)} .
$$


The term $S_{\nu}(t)$ is a Riemann sum corresponding to the integral

$$
\int_{0}^{t} f(\tau) d \tau, \quad f(\tau):=\tau^{r} \cos \tau
$$

and the subdivision

$$
0<\frac{t}{\nu}<\cdots<\frac{(\nu-1) t}{\nu}<t
$$

of the interval $[0, t]$. A simple application of the mean value theorem implies that there exist points

$$
\theta_{m} \in\left[\frac{(m-1) t}{\nu}, \frac{m t}{\nu}\right]
$$

such that

$$
\int_{0}^{t} f(\tau) d \tau=\sum_{m=1}^{\nu} f\left(\theta_{m}\right) \frac{t}{m}
$$

We deduce that

$$
\int_{0}^{t} f(\tau) d \tau-S_{\nu}(t)=\frac{t}{\nu} \sum_{m=1}^{\nu}\left(f\left(\theta_{m}\right)-f\left(\frac{m t}{\nu}\right)\right) .
$$

Now set

$$
M(t):=\sup _{0 \leq \tau \leq t}\left|f^{\prime}(\tau)\right|
$$

Observe that

$$
M(t)= \begin{cases}O\left(t^{r-1}\right), & 0 \leq t \leq 1 \\ O\left(t^{r}\right), & t>1\end{cases}
$$

We deduce

$$
\left|S_{\nu}(t)-\int_{0}^{t} f(\tau) d \tau\right| \leq M(t) \cdot \frac{t^{2}}{\nu}
$$

This, proves the $A$-part of (2.6). The $B$-part is completely similar.

We need to refine the estimates (2.6). Recall that $[m]_{r}:=m(m-$ 1) $\cdots(m-r+1), r \geq 1$. We will express $C_{\nu, r}(t)$ in terms of the sums

$$
\begin{aligned}
D_{\nu, r}(t) & :=\sum_{m=1}^{\nu}[m]_{r} z^{m}=z^{r} \frac{d^{r}}{d z^{r}}\left(\sum_{k=1}^{\nu} z^{k}\right)=z^{r} \frac{d^{r}}{d z^{r}}\left(\frac{z-z^{\nu+1}}{1-z}\right) \\
& =z^{r} \frac{d^{r}}{d z^{r}}\left(\frac{1-z^{\nu+1}}{1-z}\right) .
\end{aligned}
$$


Using the classical formula, [7, §1.4, Eq. (24d)],

$$
m^{r}=\sum_{k=1}^{r} S(r, k)[m]_{k}
$$

where $S(r, k)$ are the Stirling numbers of the second kind, we deduce,

$$
C_{\nu, r}(\zeta)=\sum_{k=1}^{r} S(r, k) D_{\nu, k}(\zeta)=\sum_{k=1}^{r} S(r, k) z^{r} \frac{d^{k}}{d z^{k}}\left(\frac{1-z^{\nu+1}}{1-z}\right)
$$

Lemma 2.4. Set $\theta:=\frac{t}{2 \nu}$, and $f(\theta)=\frac{\sin \theta}{\theta}$. Then

$$
\begin{aligned}
& \frac{t^{r+1}}{\nu^{r+1}} D_{\nu, r}(t)=\boldsymbol{i}^{r} r !\left(\frac{2 \sin \left(\frac{(\nu+1) t}{2 \nu}\right)}{f(\theta)^{r+1}} \cdot e^{\frac{i(\nu+r) t}{2 \nu}}\right. \\
&\left.-e^{i t} \sum_{j=1}^{r} i^{1-j}\left(\begin{array}{c}
\nu+1 \\
j
\end{array}\right) t^{j} \cdot\left(\frac{e^{i \theta}}{f(\theta)}\right)^{r+1-j}\right) .
\end{aligned}
$$

Proof. We have

$$
\begin{aligned}
D_{\nu, r}(t) & =z^{r} \sum_{j=0}^{r}\left(\begin{array}{c}
r \\
j
\end{array}\right) \frac{d^{j}}{d z^{j}}\left(1-z^{\nu+1}\right) \frac{d^{r-j}}{d z^{r-j}}(1-z)^{-1} \\
& =r ! \frac{z^{r}\left(1-z^{\nu+1}\right)}{(1-z)^{r+1}}-\sum_{j=1}^{r}\left(\begin{array}{c}
r \\
j
\end{array}\right)[\nu+1]_{j}(r-j) ! \frac{z^{\nu+1+r-j}}{(1-z)^{1+r-j}} \\
& =r ! \frac{z^{r}\left(1-z^{\nu+1}\right)}{(1-z)^{r+1}}-z^{\nu} r ! \sum_{j=1}^{r}\left(\begin{array}{c}
\nu+1 \\
j
\end{array}\right)\left(\frac{z}{1-z}\right)^{r+1-j} .
\end{aligned}
$$

Using the identity

$$
1-e^{i \alpha}=\frac{2}{i} \sin \left(\frac{\alpha}{2}\right) e^{\frac{i \alpha}{2}}
$$

we deduce

$$
\frac{z}{1-z}=\frac{i e^{\frac{i t}{2 \nu}}}{2 \sin \left(\frac{t}{2 \nu}\right)}
$$

and

$$
D_{\nu, r}(t)=\boldsymbol{i}^{r} \frac{r !}{2^{r}} \frac{\sin \left(\frac{(\nu+1) t}{2 \nu}\right) e^{\frac{i(\nu+1+2 r) t}{2 \nu}}}{\sin ^{r+1}\left(\frac{t}{2 \nu}\right) e^{\frac{(r+1) i t}{2 \nu}}}-e^{i t} r ! \sum_{j=1}^{r} \boldsymbol{i}^{r+1-j}\left(\begin{array}{c}
\nu+1 \\
j
\end{array}\right) \frac{e^{i(r+1-j) \theta}}{(2 \sin \theta)^{r+1-j}}
$$




$$
=\boldsymbol{i}^{r} r !\left(\frac{2 \sin \left(\frac{(\nu+1) t}{2 \nu}\right)}{(2 \sin \theta)^{r+1}} \cdot e^{\frac{i(\nu+r) t}{2 \nu}}-e^{i t} \sum_{j=1}^{r} \boldsymbol{i}^{1-j}\left(\begin{array}{c}
\nu+1 \\
j
\end{array}\right)\left(\frac{e^{i \theta}}{2 \sin \theta}\right)^{1+r-j}\right) .
$$

Multiplying both sides of the above equality by $\left(\frac{t}{\nu}\right)^{r+1}$ we get $(2.9)$.

Lemma 2.4 coupled with the fact that the function $f(\theta)$ is bounded on $\left[0, \frac{\pi}{2}\right]$ yield the following estimate.

$$
\frac{t^{r+1}}{\nu^{r+1}} D_{\nu, r}(t)=O(1), \quad \forall \nu, \quad 0 \leq t \leq \pi \nu
$$

Using (2.10) and the identity $S(r, 1)=1$ in $(2.8)$ we deduce that there exists $K=K_{r}>0$ such that for any $\nu>0$ and any $t \in[0, \pi \nu]$ we have

$\left|\frac{t^{r+1}}{\nu^{r+1}}\left(C_{\nu, r}(t)-D_{\nu, r}(t)\right)\right| \leq K_{r} \sum_{j=0}^{r-1}\left|\frac{t^{r+1}}{\nu^{r+1}} D_{\nu, j}(t)\right| \leq K_{r} \sum_{j=0}^{r-1}\left(\frac{t}{\nu}\right)^{r-j} \leq K_{r} \frac{t}{\nu}$,

so that

$$
\left|\frac{1}{\nu^{r+1}}\left(C_{\nu, r}(t)-D_{\nu, r}(t)\right)\right| \leq K_{r} \frac{1}{\nu t^{r}} .
$$

Using Lemma 2.4 we deduce

$$
\lim _{\nu \rightarrow \infty} \frac{t^{r+1}}{\nu^{r+1}} \mathbf{R e} D_{\nu, r}(t)=I_{r}(t):=\boldsymbol{i}^{r} r !\left(2 \sin \left(\frac{t}{2}\right) \cdot e^{\frac{i t}{2}}-e^{i t} \sum_{j=1}^{r} \boldsymbol{i}^{1-j} \frac{t^{j}}{j !}\right) .
$$

uniformly for $t$ on compacts. The estimate (2.11) implies that

$$
\lim _{\nu \rightarrow \infty} \frac{t^{r+1}}{\nu^{r+1}} \operatorname{Re} D_{\nu, r}(t)=I_{r}(t) .
$$

We have the following crucial estimate whose proof can be found in Appendix A.

Lemma 2.5. For every $r \geq 0$ there exists $C_{r}>0$ such that for any $\nu>0$ we have

$$
\left|\frac{1}{\nu^{r+1}} D_{\nu, r}(t)-\frac{1}{t^{r+1}} I_{r}(t)\right| \leq \frac{C_{r}}{\nu} \frac{t^{r+1}-1}{t^{r}(t-1)}, \quad \forall 0<t \leq \pi \nu .
$$


Using Lemma 2.5 in (2.11) we deduce

$$
\begin{aligned}
\left|\frac{1}{\nu^{r+1}} C_{\nu, r}(t)-\frac{1}{t^{r+1}} I_{r}(t)\right| & \leq \frac{C_{r}}{\nu} \frac{t^{r+1}-1}{t^{r}(t-1)}, \quad \forall 0<t \leq \pi \nu . \\
I_{r}(t)=t^{r+1} C_{r}(t) & =\int_{0}^{t} \tau^{r} e^{i \tau} d \tau .
\end{aligned}
$$

Using (2.6) and (2.13a) we deduce that for any nonnegative integer $r$ there exists a positive constant $K=K_{r}>0$ such that

$$
\left|C_{\nu, r}(t)-C_{r}(t)\right| \leq \frac{K_{r}}{\nu} \frac{t^{r+1}-1}{t^{r}(t-1)}
$$

Coupling the above estimates with (2.6) we deduce

$$
C_{\nu, r}(t)=C_{r}(t)+O\left(\frac{1}{\nu}\right), \forall 0 \leq t \leq \nu,
$$

where the constant implied by the symbol $O$ depends on $k$, but it is independent of $\nu$. The last equality coupled with (2.5) implies that

$$
R_{\nu}^{(k)}(t)=R_{\infty}^{(k)}(t)+O\left(\frac{1}{\nu}\right), \quad \forall 0 \leq t \leq \nu .
$$

We deduce that, for any $t>0$ we have

$$
\lim _{\nu \rightarrow \infty} f_{\nu}(t)=f_{\infty}(t)
$$

where $f_{\nu}$ is the function defined in (2.2), while

$$
f_{\infty}(t)=\frac{\left(\bar{\lambda}_{0}^{2}-R_{\infty}^{2}\right) \bar{\lambda}_{2}-\bar{\lambda}_{0}\left(R_{\infty}^{\prime}\right)^{2}}{\left(\bar{\lambda}_{0}^{2}-R_{\infty}^{2}\right)^{\frac{3}{2}}}\left(\sqrt{1-\rho_{\infty}^{2}}+\rho_{\infty} \arcsin \rho_{\infty}\right)
$$

where

$$
\begin{gathered}
\bar{\lambda}_{0}=\lambda_{0}(\infty)=\lim _{\nu \rightarrow \infty} \lambda_{0}(\nu)=\frac{1}{3}, \quad \bar{\lambda}_{2}=\lim _{\nu \rightarrow \infty} \lambda_{2}(\nu)=\frac{1}{5}, \\
\rho_{\infty}(t)=\lim _{\nu \rightarrow \infty} \rho_{\nu}(t)=\frac{R_{\infty}^{\prime \prime}\left(\bar{\lambda}_{0}^{2}-R_{\infty}^{2}\right)+\left(R_{\infty}^{\prime}\right)^{2} R_{\infty}}{\left(\bar{\lambda}_{0}^{2}-R_{\infty}^{2}\right) \bar{\lambda}_{2}-\bar{\lambda}_{0}\left(R_{\infty}^{\prime}\right)^{2}} .
\end{gathered}
$$

We have the following result whose proof can be found in Appendix A. 
Lemma 2.6.

$$
\left|R_{\infty}(t)\right|<R_{\infty}(0),\left|R_{\infty}^{\prime \prime}(t)\right|<\left|R_{\infty}^{\prime \prime}(0)\right|, \quad \forall t>0,
$$

$$
R_{\infty}(t), \quad R_{\infty}^{\prime}(t), \quad R_{\infty}^{\prime \prime}(t)=O\left(\frac{1}{t}\right) \quad \text { as } t \rightarrow \infty
$$

$$
\left(\bar{\lambda}_{0}^{2}-R_{\infty}^{2}\right) \bar{\lambda}_{2}-\bar{\lambda}_{0}\left(R_{\infty}^{\prime}\right)^{2}>0, \quad \forall t>0 .
$$

$$
\begin{aligned}
& R_{\infty}(t)=\frac{1}{3}-\frac{1}{10} t^{2}+\frac{1}{168} t^{4}+O\left(t^{6}\right), \\
& R_{\infty}^{\prime}(t)=-\frac{1}{5} t+\frac{1}{42} t^{3}+O\left(t^{5}\right), \text { as } t \rightarrow 0 .
\end{aligned}
$$

We set

$$
\delta(t):=\max (t, 1), \quad t \geq 0 .
$$

We find it convenient to introduce new functions

$G_{\nu}(t):=\frac{1}{R_{\nu}(0)} R_{\nu}(t)=\frac{1}{\lambda_{0}(\nu)} R_{\nu}(t), \quad H_{\nu}(t)=\frac{1}{R_{\nu}^{\prime \prime}(0)} R_{\nu}^{\prime \prime}(t)=-\frac{1}{\lambda_{2}(\nu)} R_{\nu}^{\prime \prime}(t)$.

Using these notations we can rewrite (2.18c) as

$$
\underbrace{\left(1-G_{\infty}^{2}\right)-\frac{\bar{\lambda}_{0}}{\bar{\lambda}_{2}}\left(G_{\infty}^{\prime}\right)^{2}}_{=: \eta(t)}>0, \quad \forall t>0
$$

The equalities (2.18d) imply that

$$
\eta(t)=\frac{3}{4375} t^{4}+O\left(t^{6}\right), \quad \forall|t| \ll 1 .
$$

Then

$$
f_{\nu}(t)=\frac{\lambda_{2}(\nu)}{\lambda_{0}(\nu)} \times \mathcal{C}_{\nu}(t) \times\left(\sqrt{1-\rho_{\nu}^{2}}+\rho_{\nu} \arcsin \rho_{\nu}\right),
$$

where

$$
\mathcal{C}_{\nu}(t):=\frac{\left(1-G_{\nu}(t)^{2}\right)-\frac{\lambda_{0}(\nu)}{\left.\lambda_{2}(\nu)\right)}\left(G_{\nu}^{\prime}(t)\right)^{2}}{\left(1-G_{\nu}(t)^{2}\right)^{3 / 2}}
$$

and

$$
\rho_{\nu}=\frac{R_{\nu}^{\prime \prime}\left(\lambda_{0}(\nu)^{2}-R_{\nu}^{2}\right)+\left(R_{\nu}^{\prime}\right)^{2} R_{\nu}}{\left(\lambda_{0}^{2}(\nu)-R_{\nu}^{2}\right) \lambda_{2}(\nu)-\lambda_{0}\left(R_{\nu}^{\prime}\right)^{2}}=\frac{-H_{\nu}\left(1-G_{\nu}^{2}\right)+\frac{\lambda_{0}(\nu)}{\lambda_{2}(\nu)}\left(G_{\nu}^{\prime}\right)^{2} G_{\nu}}{\left(1-G_{\nu}^{2}\right)-\frac{\lambda_{0}(\nu)}{\lambda_{2}(\nu)}\left(G_{\nu}^{\prime}\right)^{2}} .
$$


Lemma 2.7. Let $\kappa \in(0,1)$. Then

$$
\mathrm{C}_{\nu}(t)=O(t), \quad \forall 0 \leq t \leq \nu^{-\kappa}
$$

where the constant implied by $O$-symbol is independent of $\nu$ and $t \in\left[0, \nu^{-\kappa}\right)$, but it could depend on $\kappa$.

Proof. Observe that for $t \in\left[0, \nu^{-\kappa}\right]$ we have

$$
G_{\nu}(t)=1-\frac{\lambda_{2}(\nu)}{2 \lambda_{0}(\nu)} t^{2}+O\left(t^{4}\right), \quad G_{\nu}^{\prime}(t)=-\frac{\lambda_{2}(\nu)}{\lambda_{0}(\nu)} t+O\left(t^{3}\right)
$$

so that

$$
\left(1-G_{\nu}(t)^{2}\right)^{-3 / 2}=\left(\frac{\lambda_{2}(\nu)}{\lambda_{0}(\nu)} t\right)^{-3} \times(1+O(t)),
$$

and

$$
\left(1-G_{\nu}(t)^{2}\right)-\frac{\lambda_{0}(\nu}{\lambda_{2}(\nu)}\left(G_{\nu}^{\prime}\right)=O\left(t^{4}\right)
$$

so that $\mathcal{C}_{\nu}(t)=O(t)$.

Lemma 2.8. Let $\kappa \in(0,1)$,

$$
\eta(t):=\left(1-G_{\nu}^{2}\right)-\frac{\bar{\lambda}_{0}}{\bar{\lambda}_{2}}\left(G_{\nu}^{\prime}\right)^{2} \text { and } \gamma(t)=1-G_{\infty}(t)^{2} .
$$

Then as $\nu \rightarrow \infty$ and $t>\nu^{-\kappa}$ we have

$$
\mathfrak{C}_{\nu}=\mathcal{C}_{\infty} \times\left(1+O\left(\frac{\left(G_{\infty}^{\prime}\right)^{2}}{\nu \eta(t)}+\frac{1}{\nu \gamma(t) \delta(t)}+\frac{1}{\nu \delta(t) \eta(t)}+\frac{1}{\nu^{2} \eta(t)}\right)\right)
$$

and

$$
\mathcal{C}_{\nu}(t)=\mathcal{C}_{\infty}(t)+O\left(\frac{\left(G_{\infty}^{\prime}\right)^{2}}{\nu \gamma(t)^{3 / 2}}+\frac{\eta(t)}{\nu \delta(t) \gamma(t)^{3 / 2}}+\frac{1}{\nu \delta(t) \gamma(t)^{3 / 2}}+\frac{1}{\nu^{2} \gamma(t)^{3 / 2}}\right)
$$

uniformly for $t>\nu^{-\kappa}$.

Proof. Observe that

$$
G_{\nu}^{2}=\left(G_{\infty}+O\left(\frac{1}{\nu}\right)\right)^{2} \stackrel{(2.18 b)}{=} G_{\infty}^{2}+O\left(\frac{1}{\nu \delta(t)}\right),
$$


so that

$$
1-G_{\infty}(t)^{2}(t)=\left(1-G_{\infty}(t)^{2}\right)\left(1+O\left(\frac{1}{\nu \delta(t) \gamma(t)}\right)\right) .
$$

For $t>\nu^{-\kappa}$ we have

$$
\frac{1}{\nu \delta(t) \gamma(t)}=O\left(\frac{1}{\nu^{1-\kappa}}\right)=o(1) \text { uniformly in } t>\nu^{-\kappa} \text { as } \nu \rightarrow \infty \text {. }
$$

Hence

$$
\begin{aligned}
& \left(1-G_{\nu}^{2}\right)^{-3 / 2}=\left(1-G_{\infty}^{2}\right)^{-3 / 2}\left(1+O\left(\frac{1}{\nu \delta(t) \gamma(t)}\right)\right) \\
& \gamma(t):=1-G_{\infty}^{2}(t) .
\end{aligned}
$$

Next observe that

$$
\begin{aligned}
& \lambda_{0}(\nu)=\frac{B_{3}(\nu+1)}{3 \nu^{3}}=\frac{1}{3}+\nu^{-1}+O\left(\nu^{-2}\right), \\
& \lambda_{2}(\nu)=\frac{B_{5}(\nu+1)}{5 \nu^{5}}=\frac{1}{5}+\nu^{-1}+O\left(\nu^{-2}\right)
\end{aligned}
$$

and

$$
\frac{\lambda_{0}(\nu)}{\lambda_{2}(\nu)}=\frac{\frac{1}{3}+\nu^{-1}+O\left(\nu^{-2}\right)}{\frac{1}{5}+\nu^{-1}+O\left(\nu^{-2}\right)}=\frac{5}{3}-\frac{10}{3} \nu^{-1}+O\left(\nu^{-2}\right) .
$$

Using (2.23) and the above estimate we deduce

$$
\begin{aligned}
\left(1-G_{\nu}(t)^{2}\right)-\frac{\lambda_{0}(\nu)}{\left.\lambda_{2}(\nu)\right)}\left(G_{\nu}^{\prime}\right)^{2} \\
(2.26)=\underbrace{\left(1-G_{\infty}^{2}\right)-\frac{\bar{\lambda}_{0}}{\bar{\lambda}_{2}}\left(G_{\infty}^{\prime}\right)^{2}}_{\eta(t)}+\frac{10}{3 \nu}\left(G_{\infty}^{\prime}\right)^{2}+O\left(\frac{1}{\nu \delta(t)}\right)+O\left(\frac{1}{\nu^{2}}\right) \\
=\left(\left(1-G_{\infty}^{2}\right)-\frac{\bar{\lambda}_{0}}{\bar{\lambda}_{2}}\left(G_{\infty}^{\prime}\right)^{2}\right) \cdot\left(1+O\left(\frac{\left(G_{\infty}^{\prime}\right)^{2}}{\nu \eta(t)}+\frac{1}{\nu \delta(t) \eta(t)}+\frac{1}{\nu^{2} \eta(t)}\right)\right) .
\end{aligned}
$$

Using (2.24) we deduce (2.21). The estimate (2.22) follows from (2.21) by invoking the definitions of $\gamma(t)$ and $\eta(t)$. 
Lemma 2.9. Let $\kappa \in\left(0, \frac{1}{4}\right)$. Then

$$
\begin{aligned}
& \rho_{\nu}=\rho_{\infty}+O\left(\frac{1}{\nu \eta(t)}+\frac{\left(G_{\infty}^{\prime}\right)^{2}}{\nu \eta(t) \delta(t)}+\frac{1}{\nu \delta(t)^{2}}+\frac{1}{\nu \delta(t)^{2} \eta(t)}+\frac{1}{\nu^{2} \eta(t) \delta(t)}\right) \\
& \forall t>\nu^{-\kappa}
\end{aligned}
$$

where the constant implied by $O$-symbol is independent of $\nu$ and $t>\nu^{-\kappa}$, but it could depend on $\kappa$.

Proof. The estimates (2.18b), (2.18d) and (2.20) imply that for $t>\nu^{-\kappa}$ we have

$$
\frac{\left(G_{\infty}^{\prime}\right)^{2}}{\nu \eta(t)}+\frac{1}{\nu \delta(t) \eta(t)}+\frac{1}{\nu^{2} \eta(t)}=O\left(\frac{1}{\nu^{1-4 \kappa}}\right)=o(1)
$$

uniformly in $t>\nu^{-\kappa}$. We conclude from (2.26) that

$$
\begin{aligned}
& \left(\left(1-G_{\nu}(t)^{2}\right)-\frac{\lambda_{0}(\nu)}{\left.\lambda_{2}(\nu)\right)}\left(G_{\nu}^{\prime}\right)^{2}\right)^{-1}=\left(\left(1-G_{\infty}^{2}\right)-\frac{\bar{\lambda}_{0}}{\bar{\lambda}_{2}}\left(G_{\infty}^{\prime}\right)^{2}\right)^{-1} \\
& \times\left(1+O\left(\frac{\left(G_{\infty}^{\prime}\right)^{2}}{\nu \eta(t)}+\frac{1}{\nu \delta(t) \eta(t)}+\frac{1}{\nu^{2} \eta(t)}\right)\right) .
\end{aligned}
$$

Since $H_{\nu}=H_{\infty}+O\left(\nu^{-1}\right)$ we deduce

$$
-H_{\nu}\left(1-G_{\nu}\right)^{2}+\frac{\lambda_{0}(\nu)}{\lambda_{2}(\nu)}\left(G_{\nu}^{\prime}\right)^{2} G_{\nu}=-H_{\infty}\left(1-G_{\infty}\right)^{2}+\frac{\bar{\lambda}_{0}}{\bar{\lambda}_{2}}\left(G_{\infty}^{\prime}\right)^{2} G_{\infty}+O\left(\frac{1}{\nu}\right) .
$$

Recalling that

$$
\rho_{\nu}=\frac{-H_{\nu}\left(1-G_{\nu}^{2}\right)+\frac{\lambda_{0}(\nu)}{\lambda_{2}(\nu)}\left(G_{\nu}^{\prime}\right)^{2} G_{\nu}}{\left(1-G_{\nu}^{2}\right)-\frac{\lambda_{0}(\nu)}{\lambda_{2}(\nu)}\left(G_{\nu}^{\prime}\right)^{2}}
$$

and $-H_{\infty}\left(1-G_{\infty}\right)^{2}+\frac{\bar{\lambda}_{0}}{\lambda_{2}}\left(G_{\infty}^{\prime}\right)^{2} G_{\infty}=O\left(\delta^{-1}\right)$ we see that $(2.27)$ follows from (2.28).

Consider the function

$$
A(u)=\sqrt{1-u^{2}}+u \arcsin u,|u| \leq 1 .
$$

Observe that

$$
\frac{d A}{d u}=O(1), \quad \forall|u| \leq 1,
$$




$$
\frac{d A}{d u}=\arcsin u=O(u) \text { as } u \rightarrow 0 .
$$

Now fix an exponent $\kappa \in\left(0, \frac{1}{4}\right)$. We discuss separately two cases.

1. If $\nu^{k}<t<\pi \nu$. Then in this range we have

$$
\frac{1}{\delta}, \quad \rho_{\infty}=O\left(t^{-1}\right), \quad \rho_{\nu}=\rho_{\infty}+o(1), \frac{1}{\eta(t)}=O(1),
$$

and using (2.29b) and (2.27) we deduce

$$
\begin{aligned}
A\left(\rho_{\nu}\right) & =A\left(\rho_{\infty}\right)+A^{\prime}\left(\rho_{\infty}\right)\left(\rho_{\nu}-\rho_{\infty}\right)+O\left(\left(\rho_{\nu}-\rho_{\infty}\right)^{2}\right) \\
& =A\left(\rho_{\infty}\right)+O\left(\frac{1}{t \nu}+\frac{1}{\nu^{2}}\right) .
\end{aligned}
$$

2. $\nu^{-\kappa}<t<\nu^{\kappa}$. The equality (2.20) shows that in this range we have

$$
\frac{1}{\eta(t)}=O\left(\nu^{-4 \kappa}\right), \frac{1}{\delta(t)}=O(1)
$$

so that and (2.27) implies that

$$
\rho_{\nu}-\rho_{\infty}=O\left(\frac{1}{\nu^{1-4 k}}\right)
$$

Using (2.29a) we deduce

$$
A\left(\rho_{\nu}\right)-A\left(\rho_{\infty}\right)=O\left(\frac{1}{\nu^{1-4 k}}+\frac{1}{\nu^{1-4 \kappa} \delta(t)^{2}}+\frac{1}{\nu^{2-8 \kappa} \delta(t)}\right) .
$$

Set

$$
\Delta_{\nu}:=\mathcal{C}_{\nu} A\left(\rho_{\nu}\right)-\mathcal{C}_{\infty} A\left(\rho_{\infty}\right), \quad q_{\nu}:=\left(\frac{\lambda_{2}(\nu)}{\lambda_{0}(\nu)}\right), \quad q_{\infty}=\left(\frac{\bar{\lambda}_{2}}{\bar{\lambda}_{0}}\right)
$$

Then using (2.25) we deduce that

$$
q_{\nu}-q_{\infty}=\frac{6}{5} \nu^{-1}+O\left(\nu^{-2}\right), \quad q_{\nu}=q_{\infty}\left(1+2 \nu^{-1}+O\left(\nu^{-2}\right)\right) .
$$

Then

$$
\left(f_{\nu}(t)-q_{\nu}\right)-\left(f_{\infty}(t)-q_{\infty}\right)=
$$




$$
\begin{gathered}
=q_{\nu}\left(\mathcal{C}_{\infty} A_{\infty}\left(\rho_{\infty}\right)-1+\Delta_{\nu}\right)-q_{\infty}\left(\mathcal{C}_{\infty} A\left(\rho_{\infty}\right)-1\right) \\
=\left(q_{\nu}-q_{\infty}\right)\left(\mathcal{C}_{\infty} A\left(\rho_{\infty}\right)-1\right)+q_{\infty} \Delta_{\nu} .
\end{gathered}
$$

To prove (1.2) we need to prove the following equality.

$$
\begin{aligned}
& \left(q_{\nu}-q_{\infty}\right) \int_{0}^{\pi \nu}\left(\mathcal{C}_{\infty}(t) A\left(\rho_{\infty}(t)\right)-1\right) d t \\
& q_{\infty} \int_{0}^{\pi \nu} \Delta_{\nu}(t) d t=o(1) \text { as } \nu \rightarrow \infty .
\end{aligned}
$$

We can dispense easily of the first integral above since $\mathcal{C}_{\infty}(t) A\left(\rho_{\infty}(t)\right)-1$ is absolutely integrable on $[0, \infty)$ and $q_{\nu}-q_{\infty}=O\left(\nu^{-1}\right)$.

The second integral requires a bit of work. More precisely, we will show the following result.

Lemma 2.10. If $0<\kappa<\frac{1}{5}$, then

$$
\int_{0}^{\nu^{-\kappa}} \Delta_{\nu}(t) d t, \int_{\nu^{-\kappa}}^{\nu^{\kappa}} \Delta_{\nu}(t) d t, \int_{\nu^{\kappa}}^{\pi \nu} \Delta_{\nu}(t) d t=o(1) \text { as } \nu \rightarrow \infty .
$$

Proof. We will discuss each of the three cases separately.

1. $0<t<\nu^{-\kappa}$. The easiest way to prove that $\int_{0}^{\nu^{-\kappa}} \Delta_{\nu}(t) d t \rightarrow 0$ is to show that

$$
\mathcal{C}_{\nu}(t) A\left(\rho_{\nu}(t)\right)=O(1), \quad 0<t<\nu^{-k} .
$$

This follows using Lemma 2.7 and observing that the function $A(u)$ is bounded.

2. $\nu^{-\kappa}<t<\nu^{\kappa}$. In this range we have

$$
\frac{1}{\eta}=O\left(\nu^{-4 \kappa}\right), \frac{1}{\gamma(t)}=O\left(\nu^{-2 \kappa}\right), \frac{1}{\delta(t)}=O(1), G_{\infty}^{\prime}(t)=O(1) .
$$

Using (2.22), (2.31) we deduce

$$
\mathcal{C}_{\nu}(t)=\mathcal{C}_{\infty}(t)+O\left(\frac{1}{\nu^{1-3 \kappa}}\right), A\left(\rho_{\nu}\right)=A\left(\rho_{\infty}\right)+O\left(\frac{1}{\nu^{1-4 \kappa}}\right) .
$$

Hence

$$
\Delta_{\nu}=O\left(\frac{1}{\nu^{1-4 \kappa}}\right) \text { and } \int_{\nu^{-\kappa}}^{\nu^{\kappa}} \Delta_{\nu}(t) d t=O\left(\frac{1}{\nu^{1-5 \kappa}}\right)=o(1) .
$$


3. $\nu^{\kappa}<t<\pi \nu$. For these values of $t$ we have

$$
\eta(t), \frac{1}{\eta(t)}=O(1), \frac{1}{\delta(t)}=O\left(t^{-1}\right) .
$$

Using (2.22) and (2.30) we deduce

$$
\mathcal{C}_{\nu}(t)=\mathcal{C}_{\infty}(t)+O\left(\frac{1}{\nu t}+\frac{1}{\nu^{2}}\right), A\left(\rho_{\nu}\right)=A\left(\rho_{\infty}\right)+O\left(\frac{1}{\nu t}+\frac{1}{\nu^{2}}\right)
$$

so that

$$
\Delta_{\nu}(t)=O\left(\frac{1}{\nu t}+\frac{1}{\nu^{2}}\right), \quad \int_{\nu^{-\kappa}}^{\pi \nu} \Delta_{\nu}(t) d t=O\left(\frac{1}{\nu}+\frac{\log \nu}{\nu}\right)=o(1) .
$$

The fact that $\delta_{\infty}$ defined as in (1.2) is positive follows by arguing exactly as in $[4, \S 3.2]$. This completes the proof of Theorem 1.1.

Remark 2.11. The proof of Lemma 2.10 shows that for any $\varepsilon>0$ we have

$$
\operatorname{var}\left(Z_{\nu}\right)=\nu \delta_{\infty}+O\left(\nu^{\varepsilon}\right) \text { as } \nu \rightarrow \infty .
$$

Numerical experiments suggest that $\delta_{\infty} \approx 0.35$.

\section{A. Some elementary estimates}

Proof of Lemma 2.1. Consider the complex valued random process

$$
\mathcal{F}_{\nu}(t):=\frac{1}{\sqrt{\pi \nu^{3}}} \sum_{m=1}^{\nu} m z_{m} e^{\frac{i m t}{\nu}}, \quad z_{m}=c_{m}-\boldsymbol{i} d_{m} .
$$

The covariance function of this process is

$$
\mathcal{R}_{\nu}(t)=\boldsymbol{E}\left(\mathcal{F}_{\nu}(t) \overline{\mathcal{F}}_{\nu}(0)\right)=\frac{1}{\pi \nu^{3}} \sum_{m=1}^{\nu} m^{2} e^{\frac{i m t}{\nu}} .
$$

Observe that $\operatorname{Re} \mathcal{F}_{\nu}=\Phi_{\nu}$. Note that the spectral measure of the process $\mathcal{F}_{\nu}$ is

$$
d \sigma_{\nu}=\frac{1}{\pi \nu^{3}} \sum_{m=1}^{\nu} m^{2} \delta \frac{m}{\nu}
$$


where $\delta_{t_{0}}$ denotes the Dirac measure on $\mathbb{R}$ concentrated at $t_{0}$. We form the covariance matrix of the Gaussian vector valued random variable

$$
\left[\begin{array}{c}
\mathcal{F}_{\nu}(0) \\
\mathcal{F}_{\nu}(t) \\
\mathcal{F}_{\nu}^{\prime}(0) \\
\mathcal{F}_{\nu}^{\prime}(t)
\end{array}\right] \longrightarrow X_{\nu}(t)=\left[\begin{array}{cccc}
\mathcal{R}_{\nu}(0) & \overline{\mathcal{R}}_{\nu}(t) & \overline{\mathcal{R}}_{\nu}^{\prime}(0) & \overline{\mathcal{R}}_{\nu}^{\prime}(t) \\
\mathcal{R}_{\nu}(t) & \mathcal{R}_{\nu}(0) & -\boldsymbol{i} \mathcal{R}_{\nu}^{\prime}(t) & -\boldsymbol{i} \overline{\mathcal{R}}_{\nu}^{\prime}(0) \\
\mathcal{R}_{\nu}^{\prime}(0) & i \overline{\mathcal{R}}_{\nu}^{\prime}(t) & -\mathcal{R}_{\nu}^{\prime \prime}(0) & -\overline{\mathcal{R}}_{\nu}^{\prime}(t) \\
\mathcal{R}_{\nu}^{\prime}(t) & \boldsymbol{i} \mathcal{R}_{\nu}^{\prime}(0) & -\mathcal{R}_{\nu}^{\prime \prime}(t) & -\mathcal{R}_{\nu}^{\prime \prime}(0)
\end{array}\right]
$$

Observe that $\boldsymbol{R e} X(t)=\boldsymbol{R e} \boldsymbol{\Xi}(t)$. If we let

$$
\vec{z}=\left[\begin{array}{l}
u_{0} \\
v_{0} \\
u_{1} \\
v_{1}
\end{array}\right] \in \mathbb{C}^{4}
$$

Then, as in [2, Eq. (10.6.1)] we have

$$
\left\langle X_{\nu} \vec{z}, \vec{z}\right\rangle=\frac{1}{\pi \nu^{3}} \sum_{m=1}^{\nu} m^{2}\left|\left(u_{0}+v_{0} e^{\frac{i m t}{\nu}}\right)+\frac{i m}{\nu}\left(u_{1}+v_{1} e^{\frac{i m t}{\nu}}\right)\right|^{2} .
$$

Hence

(A.1) $\left\langle X_{\nu} \vec{z}, \vec{z}\right\rangle=0 \Leftrightarrow\left(u_{0}+v_{0} e^{\frac{i m t}{\nu}}\right)+\frac{i m}{\nu}\left(u_{1}+v_{1} e^{\frac{i m t}{\nu}}\right)=0, \forall m=1, \ldots, \nu$.

We see that if the linear system (A.1) has a nontrivial solution $\vec{z}$, then the complex $4 \times 4$ matrix

$$
A_{\nu}(t):=\left[\begin{array}{cccc}
1 & \zeta & 1 & \zeta \\
1 & \zeta^{2} & 2 & 2 \zeta^{2} \\
1 & \zeta^{3} & 3 & 3 \zeta^{3} \\
1 & \zeta^{4} & 4 & 4 \zeta^{4}
\end{array}\right], \zeta=e^{\frac{i t}{\nu}}
$$

must be singular, i.e., $\operatorname{det} A_{\nu}(t)=0$. We have

$$
\operatorname{det} A_{\nu}(t)=\operatorname{det}\left[\begin{array}{cccc}
1 & \zeta & 1 & \zeta \\
0 & \zeta^{2}-\zeta & 1 & 2 \zeta^{2}-z \\
0 & \zeta^{3}-\zeta & 2 & 3 \zeta^{3}-z \\
0 & \zeta^{4}-z & 3 & 4 \zeta^{4}-z
\end{array}\right]=\zeta^{2} \operatorname{det}\left[\begin{array}{cccc}
1 & 1 & 1 & 1 \\
0 & \zeta-1 & 1 & 2 \zeta-1 \\
0 & \zeta^{2}-1 & 2 & 3 \zeta^{2}-1 \\
0 & \zeta^{3}-1 & 3 & 4 \zeta^{3}-1
\end{array}\right]
$$




$$
\begin{aligned}
& =\zeta^{2} \operatorname{det}\left[\begin{array}{ccc}
\zeta-1 & 1 & 2 \zeta-1 \\
\zeta^{2}-1 & 2 & 3 \zeta^{2}-1 \\
\zeta^{3}-1 & 3 & 4 \zeta^{3}-1
\end{array}\right]=\zeta^{2} \operatorname{det}\left[\begin{array}{ccc}
\zeta-1 & 1 & \zeta \\
\zeta^{2}-1 & 2 & 2 \zeta^{2} \\
\zeta^{3}-1 & 3 & 3 \zeta^{3}
\end{array}\right] \\
& =\zeta^{3} \operatorname{det}\left[\begin{array}{ccc}
\zeta-1 & 1 & 1 \\
\zeta^{2}-1 & 2 & 2 \zeta \\
\zeta^{3}-1 & 3 & 3 \zeta^{2}
\end{array}\right]=\zeta \operatorname{det}\left[\begin{array}{ccc}
\zeta-1 & 1 & 0 \\
\zeta^{2}-1 & 2 & 2 \zeta^{2}-2 \\
\zeta^{3}-1 & 3 & 3 \zeta^{3}-3
\end{array}\right] \\
& =\zeta^{3} \operatorname{det}\left[\begin{array}{ccc}
\zeta-1 & 1 & 0 \\
\zeta^{2}-2 \zeta+1 & 0 & 2 \zeta^{2}-2 \\
\zeta^{3}-3 \zeta+2 & 0 & 3 \zeta^{3}-3
\end{array}\right] \\
& =\zeta^{3} \operatorname{det}\left[\begin{array}{ccc}
\zeta-1 & 1 & 0 \\
(\zeta-1)^{2} & 0 & 2(\zeta-1)(\zeta+1) \\
\zeta^{3}-3 \zeta+2 & 0 & 3 \zeta^{3}-3
\end{array}\right] \\
& =\zeta^{3}(\zeta-1) \operatorname{det}\left[\begin{array}{ccc}
\zeta-1 & 1 & 0 \\
(\zeta-1) & 0 & 2(\zeta+1) \\
(\zeta-1)^{2}(\zeta+1) & 0 & 3(\zeta-1)\left(\zeta^{2}+\zeta+1\right)
\end{array}\right] \\
& =\zeta^{3}(\zeta-1)^{3} \operatorname{det}\left[\begin{array}{ccc}
1 & 1 & 0 \\
1 & 0 & 2(\zeta+1) \\
\zeta+1) & 0 & 3\left(\zeta^{2}+\zeta+1\right)
\end{array}\right] \\
& =-\zeta^{3}(\zeta-1)^{3}\left(3\left(\zeta^{2}+\zeta+1\right)-2\left(\zeta^{2}+2 \zeta+1\right)\right) \\
& =-\zeta^{3}(\zeta-1)^{3}\left(\zeta^{2}-\zeta-1\right) \text {. }
\end{aligned}
$$

Since $|\zeta|=1$, we that $\operatorname{det} A_{\nu}(t)=0$ if and only if $t \in 2 \pi \nu \mathbb{Z}$.

Proof of Lemma 2.5. Recall that $\theta:=\frac{t}{2 \nu}, f(\theta):=\frac{\sin \theta}{\theta}$. By (2.9) we have

$$
\begin{aligned}
& \frac{t^{r+1}}{\boldsymbol{i}^{r} \nu^{r+1}} D_{\nu, r}(t) \\
& =r !\left(\frac{2 \sin \left(\frac{(\nu+1) t}{2 \nu}\right)}{f(\theta)^{r+1}} \cdot e^{\frac{i(\nu+r) t}{2 \nu}}-e^{i t} \sum_{j=1}^{r} \boldsymbol{i}^{1-j} \frac{\left(\begin{array}{c}
\nu+1 \\
j
\end{array}\right)}{\nu^{j}} t^{j} \cdot\left(\frac{e^{i \theta}}{f(\theta)}\right)^{r+1-j}\right) .
\end{aligned}
$$

Using (2.12) we deduce that

$$
\left.\mid \frac{t^{r+1}}{\boldsymbol{i}^{r} \nu^{r+1}} D_{\nu, r}(t)\right)-\frac{1}{\boldsymbol{i}^{r}} I_{r}(t)\left|\leq 2 r ! t^{r+1}\right| \frac{\sin \left(\frac{(\nu+1) t}{2 \nu}\right)}{f(\theta)^{r+1}} e^{i r \theta}-\sin \left(\frac{t}{2}\right) \mid
$$




$$
+r ! \sum_{j=1}^{r} t^{j}\left|\frac{\left(\begin{array}{c}
\nu+1 \\
j
\end{array}\right)}{\nu^{j}}\left(\frac{e^{i \theta}}{f(\theta)}\right)^{r+1-j}-\frac{1}{j !}\right|
$$

In the sequel we will use Landau's symbol $O$. The various implied constants will be independent of $\nu$. Throughout we assume $0<t \leq \pi \nu$. For $0<\theta<\frac{\pi}{2}$ and $0 \leq j \leq r$ we have

$$
\begin{gathered}
e^{i \theta}=1+O(\theta), \frac{\left(\begin{array}{c}
\nu+1 \\
j
\end{array}\right)}{\nu^{j}}=1+O\left(\frac{1}{\nu}\right), \sin \left(\frac{(\nu+1) t}{2 \nu}\right)=\sin \left(\frac{t}{2}\right)+O(\theta), \\
\frac{e^{i \theta}}{f(\theta)}=\frac{\theta(\cos \theta+i \sin \theta)}{\sin \theta}=1+O(\theta) .
\end{gathered}
$$

Hence

$$
\left|\frac{\sin \left(\frac{(\nu+1) t}{2 \nu}\right)}{f(\theta)^{r+1}} e^{i r \theta}-\sin \left(\frac{t}{2}\right)\right|=O(\theta)
$$

while for any $1 \leq j \leq r$ we have

$$
\left|\frac{\left(\begin{array}{c}
\nu+1 \\
j
\end{array}\right)}{\nu^{j}}\left(\frac{e^{i \theta}}{f(\theta)}\right)^{r+1-j}-\frac{1}{j !}\right|=O\left(\frac{1}{\nu}+\theta\right) .
$$

Using (A.3) and (A.4) in (A.2) we deduce that

$$
\left.\mid \frac{t^{r+1}}{\boldsymbol{i}^{r} \nu^{r+1}} D_{\nu, r}(t)\right)-\frac{1}{\boldsymbol{i}^{r}} I_{r}(t) \mid=O\left(\theta+\left(\theta+\frac{1}{\nu}\right) \sum_{j=1}^{r-1} t^{j}\right)=O\left(\frac{1}{\nu} \sum_{j=1}^{r+1} t^{j}\right) .
$$

Hence

$$
\left.\mid \frac{1}{\boldsymbol{i}^{r} \nu^{r+1}} D_{\nu, r}(t)\right)-\frac{1}{\boldsymbol{i}^{r} t^{r+1}} I_{r}(t) \mid=O\left(\frac{1}{\nu t^{r}} \frac{\left(1-t^{r+1}\right)}{(1-t)}\right) .
$$

Proof of Lemma 2.6. We have

$$
R_{\infty}^{(k)}(t)= \pm \frac{1}{t^{k+3}} \int_{0}^{t} \tau^{k+2} u(\tau) d \tau, \quad u(\tau)=\left\{\begin{array}{ll}
\sin \tau, & k \in 1+2 \mathbb{Z} \\
\cos \tau, & k \in 2 \mathbb{Z}
\end{array} .\right.
$$


Note that

$$
\left|R_{\infty}^{(k)}(0)\right|=\frac{1}{k+3}=\frac{1}{t^{k+3}} \int_{0}^{t} \tau^{k+2} d \tau .
$$

The inequality (2.18a) now follows from the inequality $|u(\tau)| \leq 1, \forall \tau$.

For any positive integer $r$ we denote by $\boldsymbol{j}_{r}$ the $r$-th jet at 0 of a onevariable function. We can rewrite (2.12) as follows:

$$
\begin{aligned}
\frac{1}{\boldsymbol{i}^{r}} I_{r}(t) & =r !\left(2 \sin \left(\frac{t}{2}\right) e^{\frac{i t}{2}}-\boldsymbol{i} e^{i t} \sum_{j=1}^{r} \frac{(-\boldsymbol{i} t)^{j}}{j !}\right) \\
& =r !\left(2 \sin \left(\frac{t}{2}\right) e^{\frac{i t}{2}}-\boldsymbol{i} e^{i t} \cdot \boldsymbol{j}_{r}\left(e^{-i t}-1\right)\right) \\
& =r !\left(2 \sin \left(\frac{t}{2}\right) e^{\frac{i t}{2}}+\boldsymbol{i} e^{i t}-\boldsymbol{i} e^{i t} \cdot \boldsymbol{j}_{r}\left(e^{-i t}\right)\right) \\
& =r !\left(-\boldsymbol{i}\left(e^{\frac{i t}{2}}-e^{-\frac{i t}{2}}\right) e^{\frac{i t}{2}}+\boldsymbol{i} e^{i t}-\boldsymbol{i} e^{i t} \cdot \boldsymbol{j}_{r}\left(e^{-i t}\right)\right) \\
& =\boldsymbol{i} r !\left(1-e^{i t} \cdot \boldsymbol{j}_{r}\left(e^{-i t}\right)\right)
\end{aligned}
$$

Hence $\operatorname{Re}\left(\frac{1}{\boldsymbol{i}^{r}} I_{r}(t)\right)=\mathbf{I m}\left(e^{i t} \cdot \boldsymbol{j}_{r}\left(e^{-i t}\right)\right)$ and $\frac{1}{t^{r+1}} I_{r}(t)=O\left(t^{-1}\right) t \rightarrow \infty$. This proves $(2.18 \mathrm{~b})$.

The spectral measure $d \sigma_{\nu}=\frac{1}{\pi \nu^{3}} \sum_{m=1}^{\nu} m^{2} \frac{m}{\nu}$ of the process $\mathcal{F}_{\nu}$ converges weakly as $\nu \rightarrow \infty$ to the measure $d \sigma_{\infty}=\frac{1}{\pi} \chi_{[0,1]} t^{2} d t$, where $\chi_{[0,1]}$ denotes the characteristic function of $[0,1]$. Indeed, an argument identical to the one used in the proof of Lemma 2.3 shows that for every continous bounded function $f: \mathbb{R} \rightarrow \mathbb{R}$ we have

$$
\lim _{\nu \rightarrow \infty} \int_{\mathbb{R}} f(t) d \sigma_{\nu}(t)=\int_{\mathbb{R}} f(t) d \sigma_{\infty}
$$

The complex valued stationary Gaussian process $\mathcal{F}_{\infty}$ on $\mathbb{R}$ with spectral measure $d \sigma_{\infty}$ has covariance function $\mathcal{R}_{\infty}=\frac{1}{\pi} \int_{0}^{1} t^{2} e^{i t} d t$. Note that $\operatorname{Re} \mathcal{R}_{\infty}=$ $R_{\infty}$. The results in $[2, \S 10.6]$ show that the covariance matrix

$$
x_{\infty}=\left[\begin{array}{cccc}
\mathcal{R}_{\infty}(0) & \overline{\mathcal{R}}_{\infty}(t) & \overline{\mathcal{R}}_{\infty}^{\prime}(0) & \overline{\mathcal{R}}_{\infty}^{\prime}(t) \\
\mathcal{R}_{\infty}(t) & \mathcal{R}_{\infty}(0) & -i \mathcal{R}_{\infty}^{\prime}(t) & -i \overline{\mathcal{R}}_{\infty}^{\prime}(0) \\
\mathcal{R}_{\infty}^{\prime}(0) & i \overline{\mathcal{R}}_{\infty}^{\prime}(t) & -\mathcal{R}_{\nu}^{\prime \prime}(0) & -\overline{\mathcal{R}}_{\infty}^{\prime}(t) \\
\mathcal{R}_{\infty}^{\prime}(t) & \boldsymbol{i} \mathcal{R}_{\infty}^{\prime}(0) & -\mathcal{R}_{\infty}^{\prime \prime}(t) & -\mathcal{R}_{\infty}^{\prime \prime}(0)
\end{array}\right]=\lim _{\nu \rightarrow \infty} x_{\nu}
$$


is nondegenerate. The equality $\operatorname{det} \boldsymbol{\operatorname { R e }} X_{\infty}(t) \neq 0, \forall t \in \mathbb{R}$ implies as in Remark 2.2 that $\mu_{\infty}(t) \neq 0,\left|\rho_{\infty}(t)\right|<1, \forall t \in \mathbb{R}$, where

$$
\mu_{\infty}=\frac{\left(\bar{\lambda}_{0}^{2}-R_{\infty}^{2}\right) \bar{\lambda}_{2}-\bar{\lambda}_{0}\left(R_{\infty}^{\prime}\right)^{2}}{\lambda_{0}^{2}-R_{\infty}^{2}}, \rho_{\infty}=\frac{R_{\infty}^{\prime \prime}\left(\bar{\lambda}_{0}^{2}-R_{\infty}^{2}\right)+\left(R_{\infty}^{\prime}\right)^{2} R_{\infty}}{\left(\bar{\lambda}_{0}^{2}-R_{\infty}^{2}\right) \bar{\lambda}_{2}-\bar{\lambda}_{0}\left(R_{\infty}^{\prime}\right)^{2}}
$$

This proves (2.18c). The equality (2.18d) follows from the Taylor expansion of $R_{\infty}$.

\section{REFERENCES}

1. Bleher, P.; Di, X. - Correlations between zeros of a random polynomial, J. Statist. Phys., 88 (1997), 269-305.

2. Cramér, H.; Leadbetter, M.R. - Stationary and Related Stochastic Processes. Sample Function Properties and Their Applications, Dover Publications, Inc., Mineola, NY, 2004.

3. DunNaGe, J.E.A - The number of real zeros of a random trigonometric polynomial, Proc. London Math. Soc., 16 (1966), 53-84.

4. Granville, A.; Wigman, I. - The distribution of zeroes of random trigonometric polynomials, Amer. J. Math., 133 (2011), 295-357.

http://front.math.ucdavis.edu/0809.1848

5. NiCOlAesCU, L.I. - Critical sets of random smooth functions on compact manifolds, http://www.nd.edu/ Inicolae/CritSetStat.pdf, arXiv: 1101.5990.

6. QuALLs, C. - On the number of zeros of a stationary Gaussian random polynomial, J. London Math. Soc., 2 (1970), 216-220.

7. Stanley, R.P. - Enumerative Combinatorics, Volume I, Cambridge Studies in Advanced Mathematics, 49, Cambridge University Press, Cambridge, 1997. 